\title{
Mémento pour la recherche avec l'être humain
}

\section{Michelle Salathé}

Lic. iur., MAE, Secrétaire générale adjointe ASSM

\footnotetext{
* Auteurs: Rita Bislimi, RN, MNsc, MAE, infirmière, Langenthal; Prof. Iren Bischofberger, Professeur à la Haute école de santé, Aarau; Prof. Jürgen Drewe, Médecin-chef, Hôpital universitaire de Bâle; Prof. em. Renato L. Galeazzi, St-Gall; Prof. em. Annemarie Kesselring, Berne; Prof. Christian Kind, médecin-chef à l'Hôpital des enfants, St-Gall; lic. iur. Michelle Salathé, MAE, Secrétaire générale adjointe, ASSM, Bâle; Prof. Christoph Rehmann-Sutter, Professeur en théorie et éthique à l'université de Lübeck; Prof. Dominique Sprumont, Directeur-adjoint Institut de droit de la santé,

Université de Neuchâtel.
}

Correspondance:

lic. iur. Michelle Salathé ASSM

Petersplatz 13

CH-4051 Bâle
Lorsqu'il y a quelques années, j'ai été élue membre de la commission d'éthique bâloise en tant que juriste, il m'a fallu faire des recherches pour réunir les bases nécessaires à l'évaluation de projets de recherche. À cette époque déjà, des cours étaient proposés aux membres des commissions d'éthique, mais seulement une fois par année. En participant à l'un de ces cours, je constatais en outre que les différentes commissions d'éthique divergeaient non seulement par leurs méthodes de travail, mais également - selon la législation cantonale - par leurs compétences à évaluer des projets de recherche. Même si, en tant que juriste, je suis habituée à devoir rechercher les bases juridiques importantes, un «mémento» m'aurait alors été très utile.

L'Académie Suisse des Sciences Médicales (ASSM) vient de rédiger un tel mémento. Il remplace ses directives concernant les «Expérimentations sur l'être humain», dépassées depuis un certain temps déjà et retirées en novembre 2008. Avec ce mémento, l'ASSM aimerait mettre à la disposition des chercheurs, des membres de commissions d'éthique et autres personnes intéressées des bases utiles à la planification, au déroulement et à l'évaluation de projets de recherche. En plus d'une réflexion concernant les questions éthiques, ce mémento récapitule les principales notions de base de la recherche et les conditions légales valables jusqu'à l'entrée en vigueur de la loi relative à la recherche sur l'être humain.

\section{Contenu}

1. Histoire de la recherche avec l'être humain

2. Notions et domaines

3. Conditions cadres juridiques

4. Introduction dans l'éthique de la recherche

5. Scientificité

6. Consentement informé

7. Pesée des chances et des risques et évaluation de l'acceptabilité

8. Problèmes éthiques dans la conception des études

9. Evaluation éthique d'études qui comportent des questions relevant des sciences humaines et sociales

10. Groupes de personnes particulièrement vulnérables

11. Aspects financiers

12. Confiance, confidentialité et protection des données

13. Vérification par une commission d'éthique de la recherche
Plutôt que de rédiger un texte contenant des instructions - comme dans ses directives médico-éthiques -, l'ASSM a délibérément choisi d'élaborer un mémento qui invite à la réflexion éthique sur les questions sensibles touchant à la recherche avec l'être humain et propose une orientation possible. La recherche doit toujours reposer sur une discussion autour des objectifs, des standards et des développements possibles. L'environnement et les circonstances sont en constante évolution et créent ainsi de nouveaux défis; le développement scientifique amène avec lui des nouvelles questions qui exigent de plus en plus des méthodes d'examen interdisciplinaires. C'est pourquoi, le mémento parle de la recherche au sens large du terme et intègre explicitement les méthodes de recherche des sciences sociales et humaines.

En plus des thèmes classiques de l'éthique de la recherche - comme par exemple la protection de sujets de recherche vulnérables ou la pesée de l'utilité et des risques - les critères de qualité des projets de recherche et le rôle des commissions d'éthique ont fait l'objet d'une étude approfondie dans ce mémento. Seule une recherche de qualité et de haut niveau recueillera l'approbation de la population. De même, un travail de qualité et de niveau élevé est attendu de la part des commissions d'éthique de la recherche. Pour les sujets de recherche potentiels, la commission d'éthique doit être garante de la valeur scientifique et éthique des projets de recherche. Cependant, le mémento stipule expressément que les responsabilités sont partagées: la responsabilité des chercheurs, l'évaluation indépendante des projets par les commissions d'éthique, le consentement libre et éclairé des participants et l'ancrage des procédures et critères éthiques dans le débat public sont les piliers de l'éthique de la recherche.

Le mémento a été élaboré par un collectif interdisciplinaire d'auteurs* sous la direction du Prof. em. Renato Galeazzi de St-Gall. À la fin de l'automne dernier, un premier projet de texte avait été soumis aux milieux intéressés; l'écho recueilli avait été très positif. L'Association des commissions d'éthique de la recherche sur l'être humain (ACER) soutient ce mémento et le recommande aux chercheurs et aux membres des commissions d'éthique.

Le mémento peut être commandé gratuitement au secrétariat général de l'ASSM ou téléchargé sur le site internet (www.assm.ch). Les versions française et anglaise du texte seront disponibles à partir de décembre. 